\title{
Prediction of protein structural classes for low-homology sequences based on predicted secondary structure Jian-Yi Yang* ${ }^{1}$, Zhen-Ling Peng ${ }^{2}$ and Xin Chen ${ }^{1}$
}

Addresses: ${ }^{1}$ Division of Mathematical Sciences, School of Physical and Mathematical Sciences, Nanyang Technological University, 21 Nanyang Link, Singapore, 637371 and ${ }^{2}$ Department of Mathematics, Bijie University, Guizhou, PR China, 551700

E-mail: Jian-Yi Yang* - yang0241@ntu.edu.sg; Zhen-Ling Peng - pengzhenling720@163.com; Xin Chen - chenxin@ntu.edu.sg

${ }^{*}$ Corresponding author

from The Eighth Asia Pacific Bioinformatics Conference (APBC 2010)

Bangalore, India I8-2I January 2010

Published: 18 January 2010

BMC Bioinformatics 2010, I I(SuppI I):S9 doi: I0.II86/I47I-2105-II-SI-S9

This article is available from: http://www.biomedcentral.com//47I-2 I05/I I/SI/S9

(c) 2010 Yang et al; licensee BioMed Central Ltd.

This is an open access article distributed under the terms of the Creative Commons Attribution License (http://creativecommons.org/licenses/by/2.0),

which permits unrestricted use, distribution, and reproduction in any medium, provided the original work is properly cited.

\begin{abstract}
Background: Prediction of protein structural classes $(\alpha, \beta, \alpha+\beta$ and $\alpha / \beta)$ from amino acid sequences is of great importance, as it is beneficial to study protein function, regulation and interactions. Many methods have been developed for high-homology protein sequences, and the prediction accuracies can achieve up to $90 \%$. However, for low-homology sequences whose average pairwise sequence identity lies between $20 \%$ and $40 \%$, they perform relatively poorly, yielding the prediction accuracy often below $60 \%$.

Results: We propose a new method to predict protein structural classes on the basis of features extracted from the predicted secondary structures of proteins rather than directly from their amino acid sequences. It first uses PSIPRED to predict the secondary structure for each protein sequence. Then, the chaos game representation is employed to represent the predicted secondary structure as two time series, from which we generate a comprehensive set of 24 features using recurrence quantification analysis, K-string based information entropy and segment-based analysis. The resulting feature vectors are finally fed into a simple yet powerful Fisher's discriminant algorithm for the prediction of protein structural classes. We tested the proposed method on three benchmark datasets in low homology and achieved the overall prediction accuracies of $82.9 \%$, $83.1 \%$ and $81.3 \%$, respectively. Comparisons with ten existing methods showed that our method consistently performs better for all the tested datasets and the overall accuracy improvements range from $2.3 \%$ to $27.5 \%$. A web server that implements the proposed method is freely available at http://wwwl.spms.ntu.edu.sg/ chenxin/RKS_PPSCl.

Conclusion: The high prediction accuracy achieved by our proposed method is attributed to the design of a comprehensive feature set on the predicted secondary structure sequences, which is capable of characterizing the sequence order information, local interactions of the secondary structural elements, and spacial arrangements of $\alpha$ helices and $\beta$ strands. Thus, it is a valuable method to predict protein structural classes particularly for low-homology amino acid sequences.
\end{abstract}




\section{Background}

The biological function of a protein is essentially associated with its tertiary structure, which is believed to be determined by its amino acid sequence via the process of protein folding [1]. Therefore, the prediction of protein's tertiary structure from amino acid sequences is a very important while challenging task in computational biology and proteomics. The tertiary structure can be broadly categorized into four structural classes based on the types and arrangements of their secondary structural elements [2]. They are the $\alpha$ class in which proteins contain mainly helices, the $\beta$ class containing mainly strands, and the other two classes with a mixture of $\alpha$ helices and $\beta$ strands the $\alpha+\beta$ class having $\beta$ strands mainly antiparallel and the $\alpha / \beta$ class having $\beta$ strands mainly parallel. It is of great value to predict protein structural classes as it is beneficial to study protein function, regulation and interactions. For instance, the searching scope of conformation will be significantly reduced for proteins whose structural classes are known [3].

A number of methods have been proposed to predict protein structural classes from amino acid sequences [4-11]. They mainly differ in the selection of feature sets used for prediction. The most common features are on the basis of the amino acid composition, which generally represent a protein as a twenty-dimensional vector corresponding to the frequencies of twenty amino acids in a given protein amino acid sequence $[4,7,8]$. However, these features ignored the important sequence order information which has been shown beneficial to the predictions. To overcome this limitation, various new features were developed on the basis of a so-called pseudo amino acid (PseAA) composition [12], and have been shown very successful in the prediction of protein structural classes $[13,14]$, especially for high-homology protein datasets. However, when low-homology datasets with pairwise sequence identity below $40 \%$ were tested, these methods were not effective any more. For instance, for the widely used dataset $25 P D B$ whose sequence homology is about $25 \%$, the reported overall accuracies with these methods were about $60 \%$ only $[5,6]$. Recently, Kurgan et al. $[15,16]$ proposed to extract features from the predicted secondary structure content rather than directly from the protein's amino acid sequence, and reported that the higher prediction accuracy can be consequently achieved, for instance, the overall accuracy of $79.7 \%$ for the dataset $25 P D B[16]$.

In this study, we would like to introduce a new comprehensive feature set that was also constructed from the predicted secondary structure, and demonstrate by experiments on three benchmark datasets that the prediction of protein structural classes can be further improved for low-homology amino acid sequences.

\section{Results and Discussion \\ The proposed method}

In the first step, we use the tool PSIPRED to predict the protein secondary structure for an amino acid sequence of interest. Then, the chaos game representation is employed to represent a predicted secondary structure as two time series, from which we generate a comprehensive set of 24 features using recurrence quantification analysis, K-string based information entropy and segmentbased analysis. The recurrence quantification analysis aims to capture the sequence order information of the time series [17], the $K$-string based information entropy to reflect certain local interactions along the secondary structure [18], and the segment-based analysis to characterize the spacial arrangements of $\alpha$ helices and $\beta$ strands (which is mainly used to differentiate between the $\alpha+\beta$ and $\alpha / \beta$ classes). Finally, the resulting $24-$ dimensional feature vector is fed into a simple yet powerful Fisher's discriminant algorithm [19] to make prediction of its protein structural class. Please see the section Methods for the details on the feature construction. A web server that implements the proposed method is freely available at [20].

\section{Prediction accuracies for three benchmark datasets}

The proposed method is tested on three benchmark datasets in low homology, including 25PDB that comprises 1673 proteins of about $25 \%$ sequence identity, 640 that comprises 640 proteins of about $25 \%$ sequence identity and 1189 that comprises 1092 proteins of about $40 \%$ sequence identity. The resulting prediction accuracies are listed in Table 1 . It can be seen that the overall accuracies for the three datasets are all above $80 \%$. To be specific, the overall accuracies of $82.9 \%, 83.1 \%$ and $81.3 \%$ are achieved for the datasets $25 P D B, 640$ and 1189, respectively. If comparing the four structural classes to each other, the predictions of proteins in the $\alpha$ classes are always the best (with accuracies about $90 \%$ for all the datasets).

We also obtained satisfactory prediction accuracies (about $85 \%$ ) for proteins in the $\beta$ and $\alpha / \beta$ classes. However, it seems very challenging to predict proteins in $\alpha+\beta$ classes as their prediction accuracies are relatively low (ranging between $65.6 \%$ and $71.4 \%$ ) when compared with the other classes. As a previous study pointed out [21], the low prediction accuracy of the $\alpha+\beta$ class might be due to its non-negligible overlap with the other classes.

\section{Comparison with existing methods}

The proposed method were compared with ten existing methods [5-7,14-17,21-23], and the experimental results are listed in Table 1. Except for the method SCPRED [16], 
Table I: Prediction accuracies of our method for three datasets and comparison with other reported results

\begin{tabular}{llccccc}
\hline Dataset & Reference & \multicolumn{6}{c}{ Prediction accuracy (\%) } \\
\hline \multirow{4}{*}{25 PDB } & {$[6]$} & $\alpha$ & $\beta$ & $\alpha+\beta$ & $\alpha / \beta$ & Overall \\
& {$[22]$} & 69.1 & 61.6 & 60.1 & 38.3 & 57.1 \\
& {$[5]$} & 60.6 & 60.7 & 44.3 & 67.9 & 58.6 \\
& {$[15]$} & NA & NA & NA & NA & 59.9 \\
& {$[17]$} & 77.4 & 66.4 & 45.4 & 61.3 & 62.7 \\
& {$[16]$} & 64.3 & 65.0 & 61.7 & 65.0 & 64.0 \\
& This paper & $\mathbf{9 3 . 7}$ & 81.3 & $\mathbf{7 1 . 7}$ & 73.1 & 80.3 \\
640 & {$[21]$} & 73.8 & $\mathbf{8 3 . 3}$ & 70.1 & $\mathbf{8 5 . 8}$ & $\mathbf{8 2 . 9}$ \\
& {$[16]$} & $\mathbf{9 0 . 6}$ & 81.0 & 33.9 & 81.9 & 62.3 \\
& This paper & 89.1 & $\mathbf{8 5 . 1}$ & $\mathbf{7 1 . 4}$ & $\mathbf{8 8 . 1}$ & $\mathbf{8 3 . 1}$ \\
& {$[7]$} & $\mathrm{NA}$ & $\mathrm{NA}$ & $\mathrm{NA}$ & $\mathrm{NA}$ & 53.8 \\
& {$[6]$} & 57.0 & 62.9 & 25.3 & 64.6 & 53.9 \\
& {$[23]$} & $\mathrm{NA}$ & $\mathrm{NA}$ & $\mathrm{NA}$ & $\mathrm{NA}$ & 54.7 \\
& {$[14]$} & 48.9 & 59.5 & 26.6 & 81.7 & 56.9 \\
& {$[5]$} & $\mathrm{NA}$ & $\mathrm{NA}$ & $\mathrm{NA}$ & $\mathrm{NA}$ & 58.9 \\
& {$[17]$} & 62.3 & 67.7 & 63.1 & 66.5 & 65.2 \\
& {$[21]$} & 75.8 & 75.2 & 31.8 & 82.6 & 67.6 \\
& {$[16]$} & 87.4 & 84.7 & 53.1 & $\mathbf{8 4 . 7}$ & 78.3 \\
& This paper & $\mathbf{8 9 . 2}$ & $\mathbf{8 6 . 7}$ & $\mathbf{6 5 . 6}$ & 82.6 & $\mathbf{8 1 . 3}$
\end{tabular}

The accuracies are evaluated by jackknife test and measured by the percentage of correctly predicted Proteins. The best results are highlighted in bold face.

the listed accuracy values are taken directly from their respective references. Because some inconsistencies were found between our test dataset 25PDB and the one used in [16] to test SCPRED (see Methods), the direct comparison with the accuracy values reported in [16] would not be fair. Therefore, to ensure a fair comparison, we re-implemented the method SCPRED by following the details presented in the reference paper, trained its classifier with the same version of PSIPRED used for our method, and then applied it to our test dataset 25PDB. It turns out that, the obtained accuracy values (by the jackknife test) for predicting proteins in the $\alpha, \beta, \alpha+\beta$ and $\alpha / \beta$ classes are $93.7 \%, 81.3 \%, 71.7 \%$ and $73.1 \%$, respectively, giving rise to the improvements of $1.1 \%$ $1.2 \%$ and $0.7 \%$ for the first three classes over those given in the reference paper. The overall prediction accuracy hence increases by $0.6 \%$ with our test dataset as well. These new accuracy values are listed in Table 1 , and we use them as the performance measurements of the method SCPRED for comparison.

From Table 1, we can see that the proposed method achieved the highest overall prediction accuracies among all the tested methods. By compared to the second highest accuracy values that were obtained with the method SCPRED, there are improvements of $2.6 \%$, $2.3 \%$, and $3 \%$ for the three test datasets, respectively. We also notice that significant improvements were made in particular for the $\alpha+\beta$ class and the $\alpha / \beta$ class. For example, the proposed method obtained the $85.8 \%$ accuracy for predicting proteins of the $\alpha / \beta$ class from the dataset $25 P D B$, which is $12.7 \%$ higher than that given by the method SCPRED. When the dataset 1189 is tested, the accuracy for predicting proteins of the $\alpha+\beta$ class is $12.5 \%$ higher than that given by the method SCPRED. Bear in mind that both SCPRED and our proposed method use features that are extracted from the secondary structure predicted with PSIPRED. The prediction improvements hence clearly indicate that our features are more comprehensive and informative than those used by SCPRED.

\section{Contribution of features}

To represent a protein, we used three different approaches to extract features from the predicted secondary structure sequences - recurrence quantification analysis, $K$-string based information entropy, and segment-based analysis. For brevity, let $R, K$ and $S$ denote the feature subsets generated by these three approaches, respectively. Below, we investigate how these feature subsets contribute to the prediction results.

Table 2 lists the overall prediction accuracies that were obtained with all the possible combinations of feature subsets. It can be seen that when the feature subsets are used individually, the resulting overall prediction accuracies for three datasets are all well above $25 \%$. It indicates that these predictions are unlikely to be random, since random assignment of protein classes generally leads to an accuracy value of about $25 \%$. In other words, every feature subset makes its own positive contributions to the predictions. On the other hand, as more features are involved in the prediction, the overall accuracy values are shown to increase steadily (The only exceptional case occurs when the feature subset $K$ is combined with the feature subset $R$, in which the accuracy value decreases slightly from $81.4 \%$ to $81.2 \%$ ). For instance, when tested on the dataset 640 , the prediction accuracy with the feature subset $R$ is $80.5 \%$. If the feature subset $K$ is added, the accuracy value increases to $81.1 \%$. If the feature subset $S$ is further added, i.e., all the extracted features are used, the accuracy value increases by another $2.0 \%$ up to $83.1 \%$. Therefore, we may conclude that these three feature

Table 2: Overall accuracies obtained with different combinations of feature subsets

\begin{tabular}{lccccccc}
\hline Dataset & $R$ & $K$ & $S$ & $R+K$ & $R+S$ & $K+S$ & $R+K+S$ \\
\hline $25 P D B$ & 81.4 & 76.0 & 72.7 & 81.2 & 82.3 & 78.2 & $\mathbf{8 2 . 9}$ \\
640 & 80.5 & 77.2 & 73.9 & 81.1 & 82.5 & 78.9 & $\mathbf{8 3 . 1}$ \\
1189 & 79.6 & 75.8 & 73.0 & 80.3 & 81.0 & 79.7 & $\mathbf{8 1 . 3}$ \\
\hline
\end{tabular}

See text for the notations of $R, K$ and $S$. 
subsets can make complementary contributions to each other to the predictions of protein structural classes.

\section{Differentiating between the $\alpha+\beta$ and $\alpha / \beta$ classes}

Because the segment-based features (i.e., the feature subset $S$ ) are aimed to mainly differentiate between the $\alpha$ $+\beta$ and $\alpha / \beta$ classes, it is very interesting to know how effective they are. To avoid any potential outside effects, we would like to make tests on (pure) datasets that comprise proteins only from the $\alpha+\beta$ and $\alpha / \beta$ classes. For this purpose, we generate a subset for each benchmark dataset by removing all the proteins in the $\alpha$ class or the $\beta$ class, and then train the classifier (i.e., Fisher's discriminant algorithm in our study) on these reduced subsets instead of the whole datasets.

Table 3 lists the prediction accuracy values obtained with the reduced subsets using different combinations of feature subsets. As we can see from the table, the combination $R+K$ provides the overall prediction accuracies that are only comparable to those given by the method SCPRED. In particular for the dataset 640, it even gives a lower accuracy value ( $82.2 \%$ v.s. $83.3 \%)$. With the addition of the feature subset $S$, the overall prediction accuracies got improved by about $3.0 \%$, and most importantly, all exceed those given by the method SCPRED. Specifically, there are the accuracy improvements of $4.3 \%, 2.6 \%$, and $4.9 \%$ for the three test datasets, respectively. These experiments further demonstrate that the segment-based features are very valuable for differentiating between the $\alpha+\beta$ and $\alpha / \beta$ classes.

\section{Conclusion}

To predict structural classes for low-homology protein sequences for which the pairwise sequence identity lies between $20 \%$ and $40 \%$, existing methods work very poorly with only relatively low accuracies obtained. In this paper, we aim to develop a new method so as to improve the prediction accuracy. To do so, we first use PSIPRED to predict the secondary structure sequence from a given amino acid sequence. Then, the chaos game representation (CGR) is employed to represent the predicted secondary structure as two time series, from which a comprehensive set of 24 features are generated by three different approaches - that is, the recurrence quantification analysis, $K$-string based information entropy, and segment-based analysis. The resulting feature vectors, each representing one protein, are fed into Fisher's discriminant algorithm for the final prediction of protein structural classes. Experimental results showed that all these features can make their own positive and complementary contributions so that higher prediction accuracies are achieved. For example, to predict structural classes of proteins in the dataset $25 P D B$, it achieved the accuracies of $92.8 \%, 83.3 \%$, $70.1 \%$ and $85.8 \%$ for the $\alpha, \beta, \alpha+\beta$ and $\alpha / \beta$ classes, respectively, and the overall accuracy of $82.9 \%$, which is $2.6 \%$ higher than that given by the state-of-the-art method SCPRED.

By comparisons with ten existing methods, we may attribute the high prediction accuracy of the proposed method to the superior performance of PSIPRED in predicting secondary structures and the comprehensive set of features that we constructed. The first attribution can be seen from the comparison with the method proposed in [15], which used the secondary structure prediction tool developed in [24] instead of PSIPRED. A previous study [25] showed that PSIPRED is superior to other competing secondary structure prediction methods. The second attribution can be seen from the comparison with the method SCPRED, which differs from our proposed method mainly in the selection of features. We used three different approaches to extract a comprehensive set of features from the predicted secondary structures, where the recurrence quantification analysis is used to capture the sequence order information of the time series, the $K$-string based information entropy to reflect certain local interactions along the secondary structure, and the segment-based features to characterize the spacial arrangements of $\alpha$ helices and $\beta$ strands. Thus, our proposed method may provide a promising tool for the accurate prediction of protein structural classes, in particular for low-homology amino acid sequences.

\section{Methods \\ Datasets}

The proposed method is tested on three low-homology protein datasets that are widely used in the literature,

Table 3: The accuracies of differentiating between the $\alpha+\beta$ and $\alpha / \beta$ classes

\begin{tabular}{|c|c|c|c|c|c|c|c|c|c|}
\hline \multirow[b]{2}{*}{ Dataset } & \multicolumn{3}{|c|}{$R+K$} & \multicolumn{3}{|c|}{$R+K+S$} & \multicolumn{3}{|c|}{ Ref. [16] } \\
\hline & $\alpha+\beta$ & $\alpha \mid \beta$ & Overall & $\alpha+\beta$ & $\alpha / \beta$ & Overall & $\alpha+\beta$ & $\alpha \mid \beta$ & Overall \\
\hline $25 P D B$ & 79.1 & 84.4 & 81.4 & 82.8 & 86.4 & 84.4 & 83.2 & 76.0 & 80.1 \\
\hline 640 & 78.4 & 85.9 & 82.2 & 83.6 & 88.1 & 85.9 & 77.2 & 89.3 & 83.3 \\
\hline 1189 & 76.8 & 83.2 & 80.5 & 81.3 & 83.8 & 82.8 & 63.1 & 88.6 & 77.9 \\
\hline
\end{tabular}

The datasets comprise only proteins in the $\alpha+\beta$ and $\alpha / \beta$ classes. 
and compared to a variety of existing methods [5-7,14-17,21-23]. The first two datasets, referred to as $25 P D B$ and 1189 respectively, are downloaded from RCSB Protein Data Bank [26] with the PDB IDs listed in the paper [6]. The dataset 25PDB contains 1673 proteins of pairwise sequence identity being about $25 \%$, whereas the dataset 1189 contains 1092 proteins of $40 \%$ sequence identity. The third protein dataset, referred to as 640, was first studied in [21]. It contains 640 proteins of $25 \%$ sequence identity and freely available from the web server at [27]. Note that the amino acid sequences in these datasets indeed represent protein domains rather than the complete proteins. Table 4 lists the numbers of proteins belonging to each structure class for the above three datasets, where protein structural classifications are retrieved from the database SCOP [28] and considered as true for prediction evaluation.

It shall be mentioned that protein sequences of the $25 P D B$ dataset are also provided at [29] by the study of [16]. However, some of them are different from those in our test dataset that were instead downloaded from the RCSB Protein Data Bank, which would not allow for a fair performance comparison if one uses the prediction accuracy values given in the paper [16]. We looked into these sequence differences, and found that our test dataset is indeed the latest version in the RCSB PDB. Therefore, we re-implemented the approach SCPRED by following the details presented in the paper [16] and tested on our test dataset. Experimental results showed that the prediction accuracies of SCPRED got further improved by $0.6 \%$ over those reported in [16].

\section{Secondary structure prediction}

Every amino acid in a protein sequence can be predicted into one of the three secondary structural elements, $\mathrm{H}$ (helix), E (strand), and C (coil). It is a problem known as protein secondary structure prediction, and many computational approaches have been developed in the past decades to predict the 3-state secondary structure from protein sequences. In this study we chose PSIPRED [30], which predicts protein secondary structure based on the position specific scoring matrices generated by PSIBLAST [31] and was shown to outperform other competing prediction methods [25]. For example, the protein 1 E0G has a domain with amino acid sequence

Table 4: The number of proteins belonging to different structural classes in the datasets

\begin{tabular}{lccccc}
\hline Dataset & $\alpha$ & $\beta$ & $\alpha / \beta$ & $\alpha+\beta$ & Total \\
\hline $25 P D B$ & 443 & 443 & 346 & 441 & 1673 \\
640 & 138 & 154 & 177 & 171 & 640 \\
1189 & 223 & 294 & 334 & 241 & 1092 \\
\hline
\end{tabular}

DSITYRVRKGDSLSSIAKRHGVNIKDVMRWNSDTANLQP

GDKLTLFVK. If we submit this sequence to the PSIPRED 2.6 web server [32], the predicted secondary structure to be returned will be CCEEEEECCСССННННННННССС НННННННСССССССССCCEEEEEEC.

Our method takes the predicted secondary structure sequence as input, but it is not tied to any specific tool for the secondary structure prediction. Any improved secondary structure prediction [33-35] would generally lead our method to higher prediction accuracy.

\section{Chaos game representation of predicted secondary structure}

Here we develop a new set of features based on the chaos game representation (CGR) of secondary structure sequences. The CGR was initially developed to visualize DNA sequences [36], and later applied to protein sequences as well $[17,37,38]$. Given a secondary structure sequence, we start with a equilateral triangle with sides of unit length and each vertex associated with a distinct letter of $\mathrm{H}, \mathrm{E}$ and $\mathrm{C}$. For each letter of the given secondary structure sequence, we then plot a point inside the triangle as follows. The first point is placed half way between the center of the triangle and the vertex corresponding to the first letter of the secondary structure sequence, and the $i$-th point is then placed half way between the $(i-1)$-th point and the vertex corresponding to the $i$-th letter. The obtained plot is then called the CGR of the secondary structure sequence. Figure 1 depicts the CGRs for four proteins, each belonging to a different structural class. It is very interesting to see that for proteins in the $\alpha$ and $\beta$ classes, the plotted points tend to be distributed around the sides $\mathrm{HC}$ and $\mathrm{EC}$, respectively. For proteins in the $\alpha+\beta$ and $\alpha / \beta$ classes, however, the points lie around both sides $\mathrm{HC}$ and EC without preference.

Observe that every secondary structure sequence gives rise to a distinct $(x, y)$-coordinate sequence of the plotted points. Hence we can faithfully model a CGR plot as a combination of two time series, one composed of the $x$ coordinates and the other of the $y$-coordinates. For simplicity, we call them the $x$-time series and $y$-time series, respectively. As we can see from Figure 2, the average values of the $x$ - and $y$-time series points for proteins in the $\alpha$-class, denoted as $\bar{x}$ and $\bar{y}$ respectively, tend to be smaller than those for proteins in the other classes. Therefore, these two quantities will be used as the first two features in our feature set to be constructed.

One might think that the above two features (i.e., $\bar{x}$ and $\bar{y}$ ) are quite similar to computing the symbol frequencies in the input secondary structural sequence, except that one is 

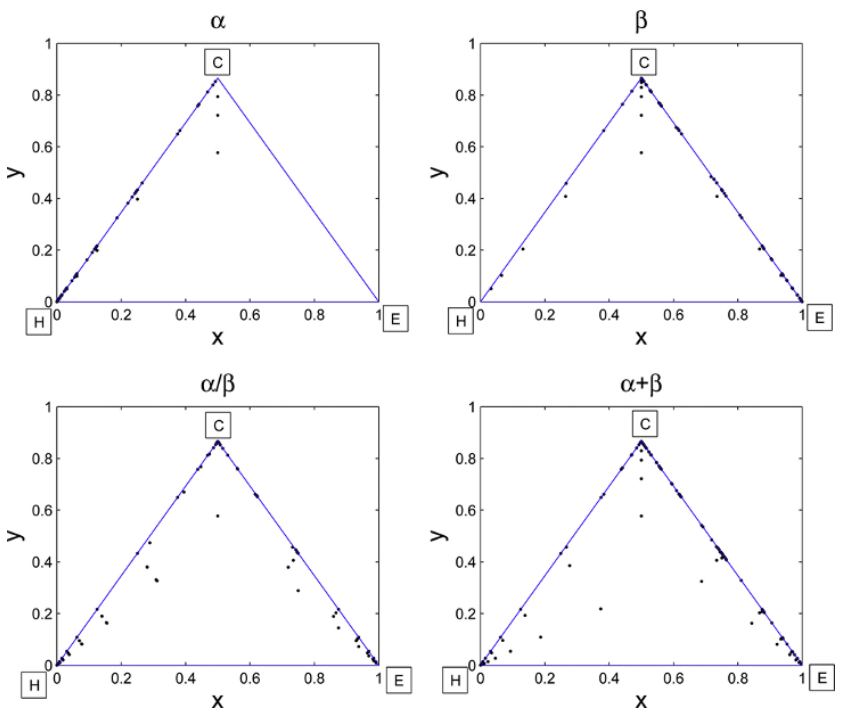

Figure I

The CGRs of predicted secondary structure for proteins from four structural classes. The blue edges represents the sides of equilateral triangles and the black points represent the CGR points. The order of the black points (corresponding to the order in the predicted secondary structure) is saved, but not shown in the figure. The PDB IDs for four proteins are IA6M (belonging to the $\alpha$ class), IAJW (belonging to the $\beta$ class), IGQOV (belonging to the $\alpha / \beta$ class), and IDEF (belonging to the $\alpha+\beta$ class).

performed in two dimension and the other in one dimension. Indeed, the features $\bar{x}$ and $\bar{y}$ take into account not only symbol frequencies but also symbol orders in the secondary structural sequence. For instance, if the input secondary structural sequence is permuted, the values of $\bar{x}$ and $\bar{y}$ will most likely change but the symbol frequencies will definitely not. It clearly demonstrates the advantage of features extracted from the chaos game representations of secondary structure sequences over those extracted directly from secondary structure sequences.

\section{Recurrence plot}

Recurrence plot (RP) is a purely graphical tool originally proposed by Eckmann et al. [39] to detect patterns of recurrence in the data. Here, we use it to describe the natural time correlation information in a time series. Given a time series $z_{1} z_{2} \ldots z_{L}$ of length $L$, we first embed it into the space $R^{m}$ of dimension $m$ using a time delay $\tau$. Let us define

$$
\left.Z_{i}=\left(z_{i}, z_{i+\tau}, z_{i+2 \tau}\right), \cdots, z_{i+(m-1) \tau}\right), \quad i=1,2, \cdots, N_{m}
$$

where $N_{m}=L-(m-1) \tau$. Hence, we obtain $N_{m}$ vectors (i.e., points) in the embedding space $R^{m}$. While the values of $m$ and $\tau$ have to be chosen appropriately based
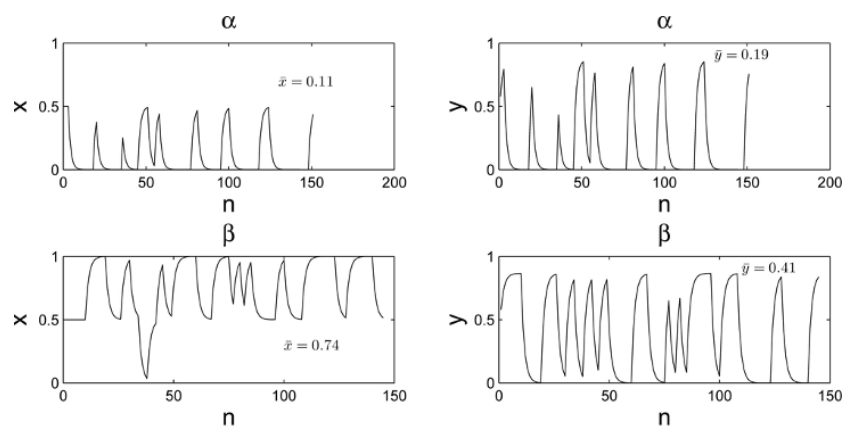

$\alpha / \beta$

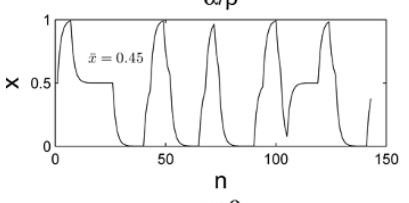

$\alpha / \beta$

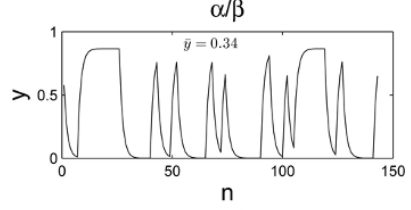

$\alpha+\beta$

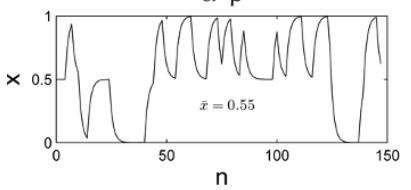

$\alpha+\beta$

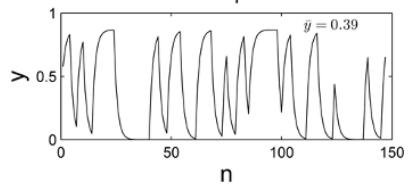

Figure 2

Eight time series that represent the four CGRs in

Figure I. Each panel in Figure I gives rise to two time series $(x$ - and $y$-coordinates, respectively). As a result, we obtain eight time series for four CGRs.

on nonlinear dynamical theory $[40], \tau$ is often set to be 1 in practice. Because an $\alpha$-helix segment generally comprises at least three residues, we set $m$ to be 3 in this study. We further construct a distance matrix (DM) of size $N_{m} \times N_{m}$ from the $N_{m}$ points, denoted as $\mathrm{DM}=\left(D_{i, j}\right)_{N_{m} \times N_{m}}$. Its elements $D_{i, j}$ are Euclidean distances between all pairs of points after scaled down by the maximum distance. As a result, all the element values of DM are located in the interval between 0 and 1 , which allows the recurrence plots in different scales to be statistically compared [40]. Finally, we define a recurrence matrix (RM) by applying a threshold $\varepsilon$ (namely radius) on the element values of DM. Formally, let $\mathrm{RM}=\left(R_{i, j}(\varepsilon)\right)_{N_{m} \times N_{m}}$ and

$$
R_{i, j}(\varepsilon)=H\left(\varepsilon-D_{i, j}\right), \quad i, j=1,2, \cdots, N_{m}
$$

where $H$ is the Heaviside function; that is, $H(x)=0$ if $x<0$, and $H(x)=1$ if $x \geq 0$.

RP is simply a visualization of RM by plotting points on $i$-j plane for those elements in RM with values equal to 1 . If $R_{i, j}(\varepsilon)=1$, we say the $j$-th point recurs with reference to the $i$-th point. For any $0<\varepsilon<1$, the RP has always a black line along main diagonal since $R_{i, i}(\varepsilon) \neq 1$. Furthermore, the RP is symmetric with respect to the main diagonal as $R_{i, j}(\varepsilon)=R_{j, i}(\varepsilon)$. For example, the RPs of the four $x$-time 

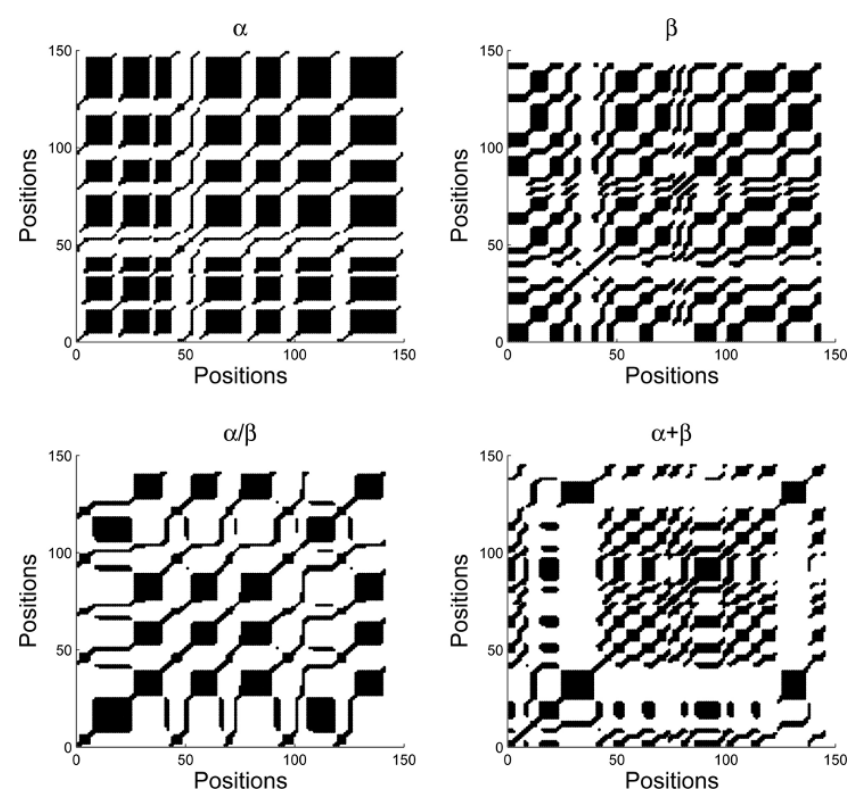

Figure 3

The corresponding RPs for the four $x$-time series in Figure 2. The parameters used are $m=3, \tau=\mathrm{I}$, and $\varepsilon=20 \%$. Note that there is a black line along the main diagonal in the plots since a point always recurs with itself. Moreover, the points in the RP are symmetric with respect to the main diagonal line.

series (and $y$-time series) of Figure 2 are shown in Figure 3 (and Figure 4, respectively). It can be seen that $\varepsilon$ is a crucial parameter in the construction of a RP. If $\varepsilon$ is chosen too small, then there might leave only a few of recurrence points so that we can not learn any recurrence structure of the underlying time series. But if $\varepsilon$ is too large, almost all the points will be enclosed in the neighbor of a point, thereby introducing a lot of structure artifacts. We will discuss the selection of the $\varepsilon$ value later in this section.

\section{Recurrence quantification analysis}

Recurrence quantification analysis (RQA) is a nonlinear technique used to quantify the information supplied by a recurrence plot $[41,42]$. In a previous study we applied the RQA to amino acid sequences for the prediction of protein structural classes [17]. Here we use it instead to analyze the predicted secondary structure sequences. Compared to 20 states (i.e., bases) of amino acid sequence, the predicted secondary structure sequences have only three states (i.e., $\mathrm{H}, \mathrm{E}$ and $\mathrm{C}$ ). By applying the recurrence quantification analysis, we obtain eight recurrence variables to characterize a predicted secondary structure sequences. The definitions of these eight recurrence variables are omitted here due to the page limit. Instead, they are provided in the Additional file 1
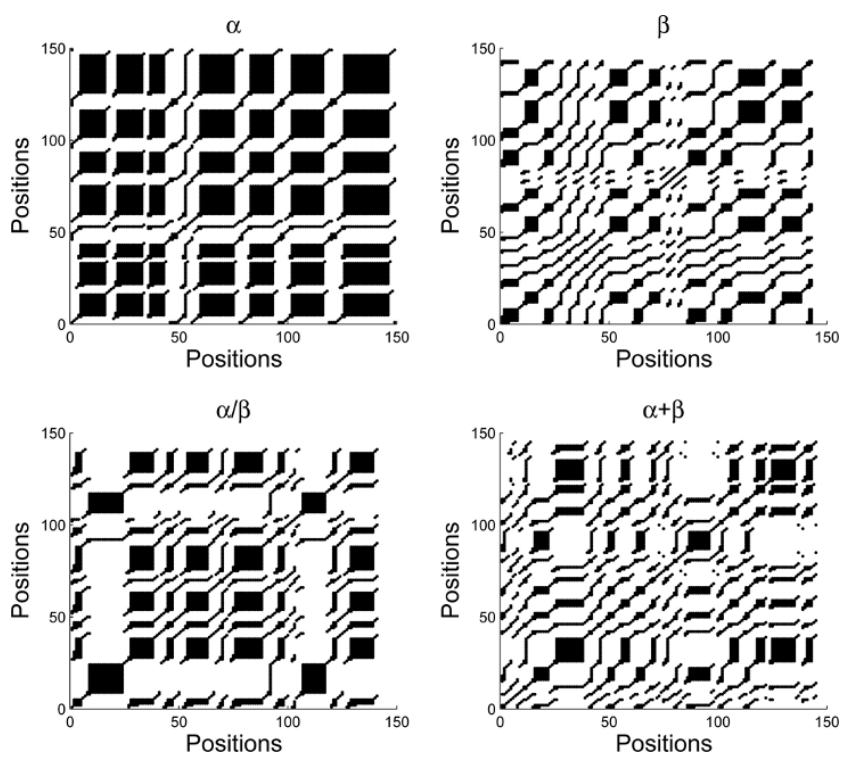

Figure 4

The corresponding RPs for the four $y$-time series in Figure 2. The parameters used are $m=3, \tau=1$, and $\varepsilon=20 \%$. Some interesting patterns can be seen to emerge from the plots, but it is not so easy to characterize them. In this study we chose the recurrence quantification analysis (RQA).

for readers' reference. These variables will be included into our set of features for the protein structural class prediction.

\section{K-string based information entropy}

Given a predicted secondary structure sequence of length $L$, we call any substring $s_{1} s_{2} \ldots s_{K}$ of length $K$ a $K$-string, where each $s_{i}$ represents a letter in the set $\{\mathrm{H}, \mathrm{E}, \mathrm{C}\}$. There are totally $3^{K}$ distinct $K$-strings for any $K$. We denote the probability of the $K$-string $s_{1} s_{2} \ldots s_{K}$ occurring in the given predicted secondary sequence by $p\left(s_{1} s_{2} \ldots\right.$ $\left.s_{K}\right)$. When $K=1, p(\mathrm{H}), p(\mathrm{E})$, and $p(\mathrm{C})$ are simply the respective probabilities of $\mathrm{H}, \mathrm{E}$ and $\mathrm{C}$ occurring in the given predicted secondary structure sequence. The $K$-th order information entropy $I_{K}$ is hence calculated as

$$
I_{K}=-\sum_{s} \sum_{s_{1} s_{2} \cdots s_{K-1}} p(s) p\left(s \mid s_{1} s_{2} \cdots s_{K-1}\right) \log _{2}\left(p\left(s \mid s_{1} s_{2} \cdots s_{K-1}\right)\right), \quad K>1,
$$

where the first sum is over the set $\{\mathrm{H}, \mathrm{E}, \mathrm{C}\}$ and the second one is over all possible $(K-1)$-strings. $p\left(s \mid s_{1} s_{2} \ldots s_{K-1}\right)$ is the conditional probability of the letter $s$ occurring after the $(K-1)$-string $s_{1} s_{2} \ldots s_{K-1}$ in the given predicted secondary structure sequence. As argued in [18], $I_{K}$ can reflect certain local interactions (i.e., correlations) along the secondary structure by using $K$-strings. 
The two quantities, $p(\mathrm{H})$ and $p(\mathrm{E})$, are included in our feature set, and have been shown very helpful in improving the prediction of protein structural classes [16]. $p(\mathrm{C})$ is not included as its value depends on $p(\mathrm{H})$ and $p(\mathrm{E})$ due to $p(\mathrm{H})+p(\mathrm{E})+p(\mathrm{C})=1$. In addition, we include $I_{2}, I_{3}, \ldots, I_{K}$ into our feature set; however, the value of $K$ remains to be determined. We will discuss this issue later in this section.

\section{Segment-based analysis}

While proteins in the $\alpha+\beta$ class and $\alpha / \beta$ class contain both $\alpha$ helices and $\beta$ strands, they differ at least in two aspects. One is the directionality of $\beta$ strands. The $\beta$ strands are mainly antiparallel in the $\alpha+\beta$ class but parallel in the $\alpha / \beta$ class. The other concerns the distributions of $\alpha$ helices and $\beta$ strands. $\alpha$ helices and $\beta$ strands are largely segregated in the $\alpha+\beta$ class, but instead largely interspersed in the $\alpha / \beta$ class. While the exact distributions can only be known from the spatial arrangement of secondary structure segments, it is still reasonable to expect that they could be more or less inferred from their secondary structure sequences. To this end, we construct below three features from the secondary structure sequences characterizing the distributions of $\alpha$ helices and $\beta$ strands, and hope that they can be used to differentiate between the $\alpha+\beta$ class from $\alpha / \beta$ class.

As the first step of feature construction, we reduce a secondary structure sequence into a segment sequence, which is composed of helix segments and strand segments (denoted by $\alpha$ and $\beta$, respectively). Here, a helix segment refers to a continuous segment of all $\mathrm{H}$ symbols in the secondary structure sequence, and a similar definition is also applied to a strand or coil segment. Since at least three and two residues are generally required to form an $\alpha$ helix segment and an $\beta$ strand segment respectively, we will ignore those helix and strand segments that do not meet this size requirement. Moreover, in order to focus on the arrangement of $\alpha$ helix and $\beta$ strand segments, the coil segments are ignored as well. For example, given a secondary structure sequence, CCEECCCHHCCHHHHEEEHHHHCCCCCCECCEECCHHHCCEEEEEEC, its reduced segment sequence is $\beta \alpha \beta \alpha \beta \alpha \beta$, in which the $\alpha$ helices and $\beta$ strands are largely interspersed, suggesting that the corresponding protein more likely belongs to the $\alpha / \beta$ class rather than $\alpha+\beta$ class.

Let $p_{t}$ denote the probability of transitions between $\alpha$ and $\beta$ segments in a segment sequence, which is essentially the relative frequency of the substring $\alpha \beta$ or $\beta \alpha$ occurring in the segment sequence. Let $p_{c_{\alpha}}$ (respectively, $p_{c_{\beta}}$ ) denote the probability of two consecutive $\alpha$ (respectively, $\beta$ ) segments. Note that $p_{t}+p_{c_{\alpha}}+p_{c_{\beta}}=1$; therefore, any probability can be deduced from the other two. In order to measure the degree of segment aggregation, we chose two of the above three probabilities to be included into our feature set. In our experimental study, $p_{t}$ and $p_{c_{\alpha}}$ are used. The third feature to be extracted is the probability of helix (or strand) segments occurring in a segment sequence, denoted by $p(\alpha)$ (or $p(\beta)$ ). Clearly, $p(\alpha)+p(\beta)=1$. $p(\beta)$ is used in this study.

\section{Prediction assessment}

As discussed above, we extract a set of $23+K-1$ features from the predicted secondary structure sequences. These feature vectors are fed into Fisher's discriminant algorithm [19] for the prediction of protein structural classes. Due to the page limit, the details of this algorithm is omitted. The prediction accuracy is measured by the proportion of proteins that are correctly predicted. The jackknife test is employed to evaluate our method. For more details about Fisher's discriminant algorithm and the prediction assessment, please refer to the Additional file 1.

\section{Selection of $\varepsilon$ and $K$}

As mentioned earlier, the value $\varepsilon$ in RQA and the number $K$ for $K$-string remain to be determined. Here, we determine their values by aiming to achieve the highest overall prediction accuracy as possible. For this purpose, a simple grid search strategy is adopted, where $\varepsilon$ is allowed to take a value only between $1 \%$ to $50 \%$ and $K$ only between 2 to 15 . We use the dataset 25PDB to compute the overall prediction accuracies for different combinations of $\varepsilon$ and $K$. For example, when $K=2$, the overall prediction accuracies for different values of $\varepsilon$ are shown in the left panel of Figure 5. When $\varepsilon=39 \%$, the overall prediction accuracies for different values of $K$ are shown in the right panel of Figure 5.
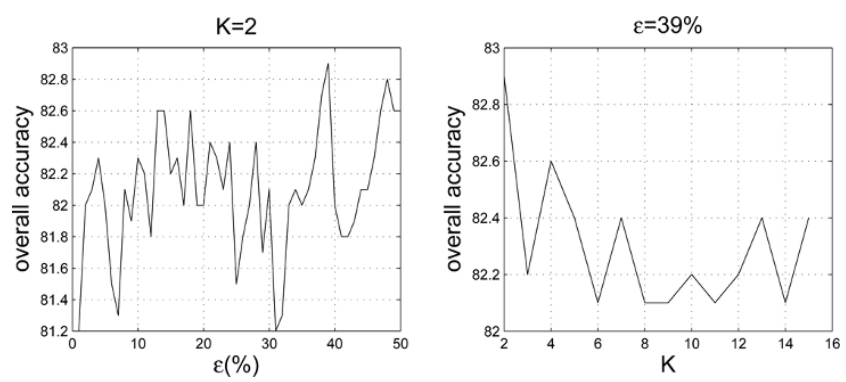

Figure 5

The overall prediction accuracies of the dataset 25PDB with varying values of $\varepsilon$ and $K$. When $K=2, \varepsilon$ ranges from $1 \%$ to $50 \%$ (left panel). When $\varepsilon=39 \%, K$ ranges between 2 and 15 (right panel). 
By the above grid search, we found that the highest accuracy $(83.0 \%)$ is obtained with the combination of $\varepsilon=48 \%$ and $K=14$ (giving rise to 36 features) and the second highest accuracy $(82.9 \%)$ is given by the combination of $\varepsilon=39 \%$ and $K=2$ (giving rise to 24 features), which is only $0.1 \%$ lower. To gain such a negligible accuracy improvement, the former combination consumes a much larger amount of computer time and memory than the latter combination when calculating feature vectors of higher dimension (i.e., 36-dimension v.s. 24-dimension). Based on this observation, we chose $\varepsilon=39 \%$ and $K=2$ in our experiments.

\section{Competing interests}

The authors declare that they have no competing interests.

\section{Authors' contributions}

JYY contributed to the conception and design of the study, downloaded the datasets, analyzed the results, has been involved in programming, drafting and revising the manuscript. ZLP has been involved in preparing the datasets, programming and discussion on the results. XC coordinated the study and has been involved in drafting and revising the manuscript. All authors read and approved the final manuscript.

\section{Additional material}

\section{Additional file 1}

The detailed descriptions about Recurrence quantification analysis, Fisher's discriminant algorithm and Prediction assessment can be found in this file.

Click here for file

[http://www.biomedcentral.com/content/supplementary/14712105-11-S1-S9-S1.pdf]

\section{Acknowledgements}

JYY would like to express his gratitude to Dr. Zu-Guo Yu for continuous encouragement and help. The authors also thank Dr. Lukasz A. Kurgan for his suggestions on the test datasets, and the anonymous referees for their valuable comments. This work was partially supported by the Singapore NRF grant NRF2007IDM-IDM002-0I0 and MOE AcRF Tier I grant RG78/ 08 .

This article has been published as part of BMC Bioinformatics Volume II Supplement 1, 2010: Selected articles from the Eighth Asia-Pacific Bioinformatics Conference (APBC 2010). The full contents of the supplement are available online at http://www.biomedcentral.com/I47I$\underline{2105 / I I \text { ? issue }=S 1 \text {. }}$.

\section{References}

I. Anfinsen C: Principles that govern the folding of protein chains. Science $\mid 973$, 181:223-230.

2. Levitt $M$ and Chothia $C$ : Structural patterns in globular proteins. Nature 1976, 261:552-558.
3. Bahar I, Atilgan AR, Jernigan RL and Erman B: Understanding the recognition of protein structural classes by amino acid composition. Proteins 1997, 29:172-185.

4. Chou KC and Zhang CT: Predicting of protein structural class. Crit Rev Biochem Mol Biol 1995, 30:275-349.

5. Kedarisetti KD, Kurgan LA and Dick S: Classifier ensembles for protein structural class prediction with varying homology. Biochem Biophys Res Commun 2006, 348:98I-988.

6. Kurgan LA and Homaeian L: Prediction of structural classes for protein sequences and domains-Impact of prediction algorithms, sequence representation and homology, and test procedures on accuracy. Pattern Recogn 2006, 39:2323-2343.

7. Wang ZX and Yuan Z: How good is the prediction of protein structural class by the component-coupled method? Pattern Recogn 2000, 38: 165-175.

8. Chou KC: A novel approach to predicting protein structural classes in a (20-I)-D amino acid composition space. Proteins 1995, 21:319-344.

9. Chou KC, Liu WM, Maggiora GM and Zhang CT: Prediction and classification of domain structural classes. Proteins 1998, 3 I: $97-130$.

10. Shen HB, Yang J, Liu XJ and Chou KC: Using supervised fuzzy clustering to predict protein structural classes. Biochem Biophys Res Commun 2005, 334:577-58I.

II. Chou KC and Maggiora GM: Domain structural class prediction. Protein Eng 1998, I I:523-538.

12. Chou KC: Prediction of protein cellular attributes using pseudo amino acid composition. Proteins 200I, 43:246-255.

13. Chen C, Tian YX, Zou XY, Cai PX and Mo JY: Using pseudoamino acid composition and support vector machine to predict protein structural class. J Theor Biol 2006, 243:444-448.

14. Zhang TL, Ding YS and Chou KC: Prediction protein structural classes with pseudo-amino acid composition: Approximate entropy and hydrophobicity pattern. J Theor Biol 2008, 250: 186-193.

15. Kurgan $L$ and Chen K: Prediction of protein structural class for the twilight zone sequences. Biochem Biophys Res Commun 2007 , 357:453-460.

16. Kurgan L, Cios K and Chen K: SCPRED: Accurate prediction of protein structural class for sequences of twilight-zone similarity with predicting sequences. BMC Bioinformatics 2008, 9:226.

17. Yang JY, Peng ZL, Yu ZG, Zhang RJ, Anh V and Wang D: Prediction of protein structural classes by recurrence quantification analysis based on chaos game representation. J Theor Biol 2009, 257:618-626.

18. Qi J, Wang B and Hao BL: Whole proteome prokaryote phylogeny without sequence alignment: a K-String composition approach. J Mol Evol 2004, 58: I-II.

19. Duda RO, Hart PE and Stork DG: Pattern Classification New York: John Wiley \& Sons; 200I.

20. RKS-PPSC web server. http://wwwl.spms.ntu.edu.sg/ chenxin/ RKS PPSCl.

21. Chen K, Kurgan LA and Ruan J: Prediction of protein structura class using novel evolutionary collocation-based sequence representation. J Comput Chem 2008, 29:1596-1604.

22. Costantini $S$ and Facchiano AM: Prediction of the protein structural class by specific peptide frequencies. Biochimie 2009, $91: 226-229$.

23. Anand A, Pugalenthi $G$ and Suganthan PN: Predicting protein structural class by SVM with class-wise optimized features and decision probabilities. J Theor Biol 2008, 253:375-380.

24. Zhang ZD, Sun ZR and Zhang CT: A new approach to predict the helix/strand content of globular proteins. J Theor Biol 200 I, 208:65-78.

25. Birzele $F$ and Kramer S: A new representation for protein secondary structure prediction based on frequent patterns. Bioinformatics 2006, 22:2628-2634.

26. RCSB Protein Data Bank. http://www.rcsb.org/pdb/home/home. do.

27. SCEC Server, http://biomine.ece.ualberta.ca.

28. Murzin A, Brenner S, Hubbard $T$ and Chothia C: SCOP: a structural classification of protein database for the investigation of sequence and structures. J Mol Biol 1995, 357: 536-540.

29. SCPRED Data. http://biomine.ece.ualberta.ca/SCPRED/SCPRED. htm.

30. Jones DT: Protein secondary structure prediction based on position specific scoring matrices. J Mol Biol 1999, 292: I 95-202. 
31. Altschul SF, Madden TL, Schäffer AA, Zhang J, Zhang Z, Miller W and Lipman DJ: Gapped BLAST and PSI-BLAST: a new generation of protein database search programs. Nucleic Acids Res 1997, 25:3389-3402.

32. PSIPRED 2.6 web server. http://bioinf.cs.ucl.ac.uk/psipred/.

33. Lin HN, Chang JM, Wu KP, Sung TY and Hsu WL: HYPROSP II-A knowledge-based hybrid method for protein secondary structure prediction based on local prediction confidence. Bioinformatics 2005, 21:3227-3233.

34. Aydin $Z$, Altunbasak $Y$ and Borodovsky M: Protein secondary structure prediction for a single-sequence using hidden semi-Markov models. BMC Bioinformatics 2006, 7:118.

35. Duan M, Huang M, Ma C, Li L and Zhou Y: Position-specific residue preference features around the ends of helices and strands and a novel strategy for the prediction of secondary structures. Protein Sci 2008, 17:1505-15/2.

36. Jeffrey $H J$ : Chaos game representation of gene structure. Nucleic Acids Res 1990, 18:2163-2170.

37. Fiser A, Tusnády GE and Simon I: Chaos game representation of protein structures. J Mol Graph 1994, I2:302-304.

38. Yu ZG, Anh V and Lau KS: Chaos game representation of protein sequences based on the detailed HP model and their multifractal and correlation analyses. I Theor Biol 2004, 226: $34 \mid-348$.

39. Eckmann JP, Kamphorst SO and Ruelle D: Recurrence plots of dynamical systems. Europhys Lett 1987, 4:973-977.

40. Riley MA and Van Orden GC: Tutorials in contemporary nonlinear methods for the behavioral sciences, Retrieved March I, 2005, from http://www.nsf.gov/sbe/bcs/pac/nmbs/ nmbs.jsp..

4I. Zbilut JP and Webber CLJ: Embeddings and delays as derived from quantification of recurrence plots. Phys Lett A 1992, I7I: 199-203.

42. Webber CLJ and Zbilut JP: Dynamical assessment of physiological systems and states using recurrence plot strategies. J Appl Physiol 1994, 76:965-973.

Publish with Biomed Central and every scientist can read your work free of charge

"BioMed Central will be the most significant development for disseminating the results of biomedical research in our lifetime. "

Sir Paul Nurse, Cancer Research UK

Your research papers will be:

- available free of charge to the entire biomedical community

- peer reviewed and published immediately upon acceptance

- cited in PubMed and archived on PubMed Central

- yours - you keep the copyright
BioMedcentral 